\title{
Using neutron-capture abundances as a tracer of Galactic halo formation
}

Cherie Fishlock*

Research School of Astronomy \& Astrophysics, Australian National University, Weston Creek ACT 2611, Australia

E-mail: cherief@mso.anu.edu.au

\section{Amanda Karakas}

Research School of Astronomy \& Astrophysics, Australian National University, Weston Creek ACT 2611, Australia

E-mail: akarakas@mso.anu.edu.au

\section{Maria Lugaro}

Monash Centre for Astrophysics, Monash University, Clayton VIC 3800, Australia

E-mail: maria.lugaro@monash.edu

\section{David Yong}

Research School of Astronomy \& Astrophysics, Australian National University, Weston Creek ACT 2611, Australia

E-mail: yong@mso.anu.edu.au

Chemical differences in stellar populations are one method to study the formation and evolution of the Galactic halo of the Milky Way. In particular, there are low $\alpha$-element stars and high $\alpha$ element stars in the Galactic halo that also show differences in their neutron-capture elemental abundances. Asymptotic giant branch (AGB) stars are an important source of neutron-capture elements that are produced via the slow $(s-)$ neutron-capture process. To better understand these abundance differences, models of AGB stars have been calculated at a metallicity of $Z=0.001$ $([\mathrm{Fe} / \mathrm{H}] \approx-1.2)$. We present theoretical abundance predictions of $\mathrm{H}$ through to $\mathrm{Pb}$ from low-mass AGB stars spanning a mass range of $1 \mathrm{M}_{\odot}$ to $3 \mathrm{M}_{\odot}$. We compare the abundance distribution of the $1.5 \mathrm{M}_{\odot}$ stellar model to that of Cristallo et al. (2011) and find that they are in good agreement. Finally, we compare final surface abundances from stellar models of $1 \mathrm{M}_{\odot}$ and $3 \mathrm{M}_{\odot}, Z=0.001$ that have been computed using two different nuclear networks. We find small abundance differences in the $s$-process peaks for the $1 \mathrm{M}_{\odot}$ but no differences in the neutron-capture elements for the $3 \mathrm{M}_{\odot}$ model.

XII International Symposium on Nuclei in the Cosmos

August 5-12, 2012

Cairns, Australia

\footnotetext{
*Speaker.
} 


\section{Introduction}

The Galactic halo of the Milky Way is believed to have formed via a combination of processes including monolithic collapse (Eggen et al. 1962) and the accretion of lower mass satellite galaxies (Searle \& Zinn 1978). The study of stellar populations, both chemically and kinematically, can reveal information about this accretion process and how it has shaped the Galactic halo we see today. Nissen \& Schuster (2010) found that the Galactic halo comprises of two stellar populations that can be chemically separated into a low- $\alpha^{1}$ population and a high- $\alpha$ population. Variations in the abundances of these populations hint at differing chemical enrichment histories. In particular, this separation into a low- $\alpha$ and high- $\alpha$ population suggests that the stars with a low- $\alpha$ abundance may have been accreted from dwarf satellite galaxies (as in the Searle \& Zinn (1978) paradigm) whereas the high- $\alpha$ population consists of stars formed in-situ in the Milky Way (as in the Eggen et al. (1962) paradigm). In a follow-up study, Nissen \& Schuster (2011) found systematic differences between the high- $\alpha$ and low- $\alpha$ halo populations for $\mathrm{Cu}, \mathrm{Zn}, \mathrm{Y}, \mathrm{Ba}$, but no differences for Mn. These authors concluded that the low- $\alpha$ population likely formed in a system with a slower chemical evolution where longer-lived low-mass stars and Type Ia supernovae had started to contribute. In comparison, the high- $\alpha$ population formed from a rapid star formation event that predominantly included the contribution from the core collapse of massive stars.

In order to study the origin of the low- $\alpha$ population we have calculated a grid of AGB model stars covering a range of mass from $1 \mathrm{M}_{\odot}$ to $6 \mathrm{M}_{\odot}$ at a metallicity of $Z=0.001([\mathrm{Fe} / \mathrm{H}] \approx-1.2)$. This metallicity was chosen because it is centred on the $[\mathrm{Fe} / \mathrm{H}]$ metallicity distribution of the low- $\alpha$ stars in the Nissen \& Schuster (2010) sample. Here we present the nucleosynthesis predictions from the low-mass end of the grid covering a range from $1 \mathrm{M}_{\odot}$ to $3 \mathrm{M}_{\odot}$. All of the stellar evolutionary models of $Z=0.001$ will be presented in detail in a forthcoming paper.

\section{Stellar modelling}

A two step procedure is performed to calculate each stellar model. First, we use the Mt Stromlo stellar evolutionary code (Karakas et al. 2010, and references therein) to calculate the stellar evolutionary sequences from the zero-age main sequence to near the end of the thermally-pulsing AGB phase. We use the same input physics (e.g., mass loss, mixing-length parameter) as in Karakas et al. (2010) with the following differences. Unlike the models discussed in Karakas et al. (2010) no convective overshoot was applied to the low-mass AGB models in order to obtain the third dredgeup. At the metallicity considered here, we find some third dredge-up in all our AGB models, even the $1 \mathrm{M}_{\odot}$. Furthermore, we have updated the opacities: We now use the OPAL opacities (Iglesias \& Rogers 1996) and low-temperature opacity tables from ÆSOPUS (Marigo \& Aringer 2009), both with a Lodders (2003) solar abundance.

Once we have calculated the stellar evolutionary sequences, we perform post-processing nucleosynthesis calculations to obtain the abundances from hydrogen through to lead using the same techniques discussed in Lugaro et al. (2012) and Karakas et al. (2012). We use a network of 320 species which comprises of isotopes from $\mathrm{H}$ to $\mathrm{Bi}$.The network is based on the JINA Reaclib ${ }^{2}$

\footnotetext{
${ }^{1}$ where $\alpha$ refers to the average abundance of $\mathrm{Mg}, \mathrm{Si}, \mathrm{Ca}$, and $\mathrm{Ti}$.

${ }^{2}$ https://groups.nscl.msu.edu/jina/reaclib/db/
} 


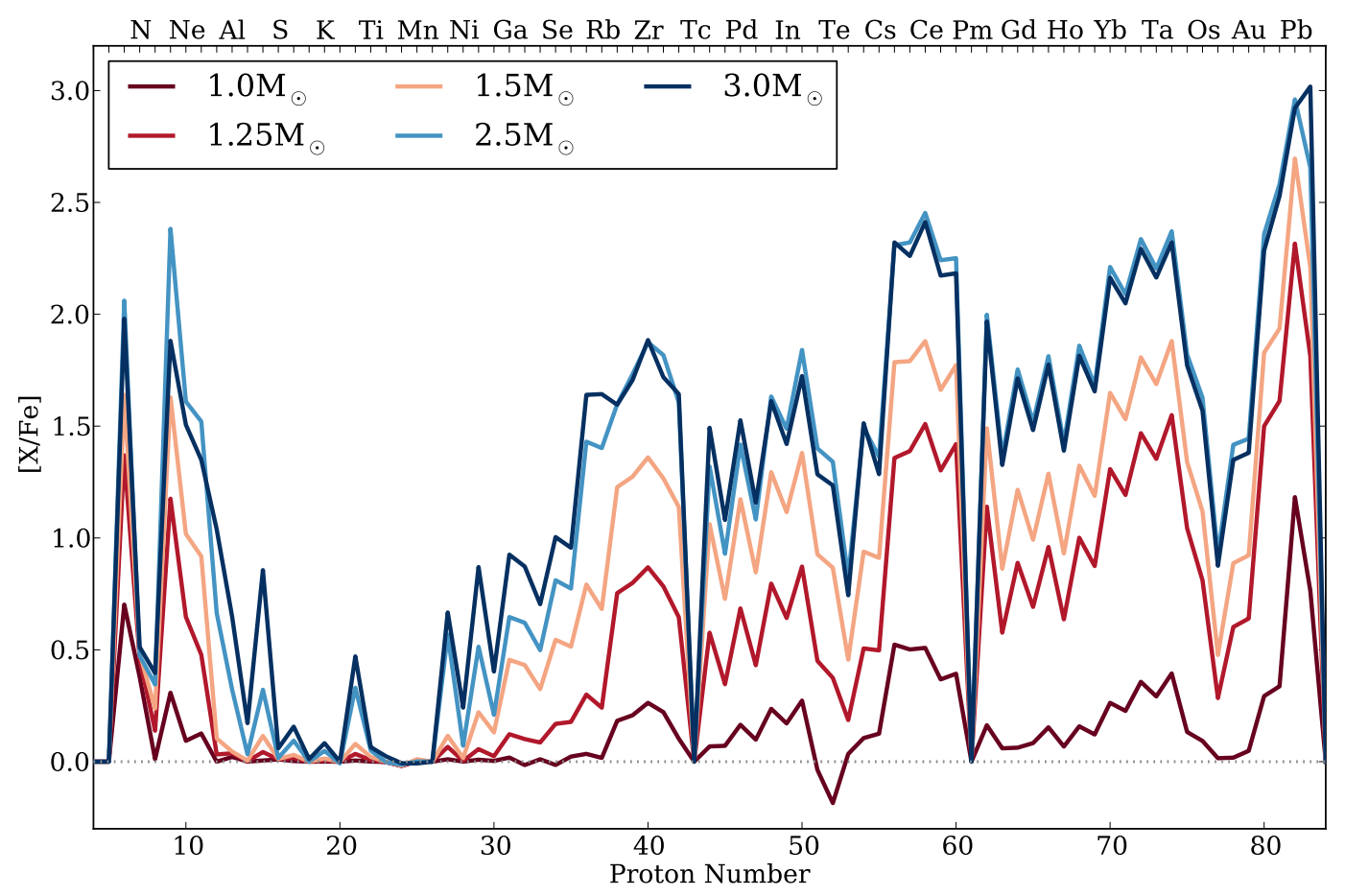

Figure 1: Final surface abundance distribution for each calculated $Z=0.001$ stellar model. $[\mathrm{X} / \mathrm{Fe}]$ represents the abundance of element $\mathrm{X}$ relative to $\mathrm{Fe}$. The abundance peaks at $\mathrm{Sr}, \mathrm{Ba}$ and $\mathrm{Pb}$ are apparent.

database from May 2009 (for more details see Lugaro et al. 2012). We use scaled solar initial abundances (based on the Asplund et al. 2009 abundances), where we set the solar $Z_{\odot}=0.015$, very close to the proto-solar nebula abundance of $Z=0.0142$ given in Asplund et al. (2009).

In the low-mass AGB models, the main neutron source is the ${ }^{13} \mathrm{C}(\alpha, \mathrm{n}){ }^{16} \mathrm{O}$ reaction (e.g., Busso et al. 1999). In order to activate this reaction efficiently, it has been shown that a ${ }^{13} \mathrm{C}$ pocket must form in the top layers of the He-intershell. The formation mechanism and extent in mass of ${ }^{13} \mathrm{C}$ pockets in AGB models is uncertain (see discussion in Herwig 2005) but it is thought that some protons mix down into the He-intershell during the deepest extent of the third dredge-up, which is when the $\mathrm{H}$-shell is inactive. Those protons are captured by the abundant ${ }^{12} \mathrm{C}$ to form ${ }^{13} \mathrm{C}$ (and also ${ }^{14} \mathrm{~N}$ depending on the number of protons) via the $\mathrm{CN}$ cycle. During the post-processing nucleosynthesis calculations, we artificially mix protons into the top $2 \times 10^{-3} \mathrm{M}_{\odot}$ layer of the Heintershell at the deepest extent of each third dredge-up episode.

\section{Results}

In Figure 1 we show the final surface abundances for each of the five models with masses from $1 \mathrm{M}_{\odot}$ to $3 \mathrm{M}_{\odot}$ at $Z=0.001$. Abundance peaks occur around $\mathrm{Sr}, \mathrm{Ba}$ and $\mathrm{Pb}$ as these elements have isotopes with a magic number of neutrons. The s-process path experiences a bottleneck at these points as the neutron-capture cross sections of magic nuclei is much lower by 2 to 3 orders of magnitude than those of the other nuclei. 


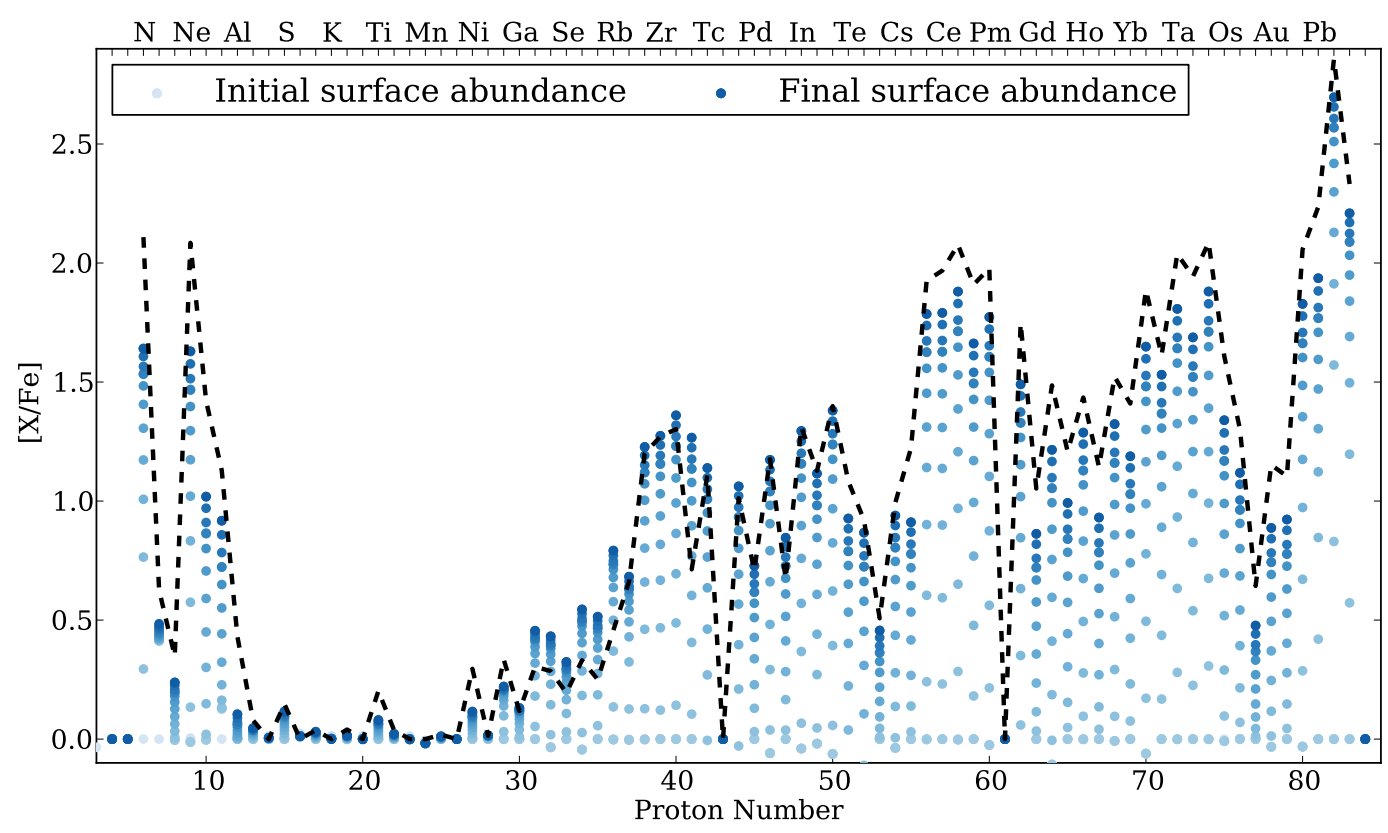

Figure 2: Surface abundances of the $1.5 \mathrm{M}_{\odot}$ stellar model of $Z=0.001$. Each point represents the surface abundance after a third dredge-up episode. The black dashed line shows the final surface abundances of a stellar model of the same mass and metallicity from Cristallo et al. (2011).

The effects of the third dredge-up on the surface composition of the $1.5 \mathrm{M}_{\odot}$ stellar model are shown in Figure 2. Each third dredge-up episode mixes material from the He-intershell, including freshly synthesised $s$-process elements, to the surface. Figure 2 also includes the results of the $1.5 \mathrm{M}_{\odot}$ stellar model of the same metallicity calculated by Cristallo et al. (2011). Their results are consistent with the final surface abundance distribution obtained using the Mt Stromlo stellar evolutionary code. For example, our final $[\mathrm{C} / \mathrm{Fe}]=1.64$, which is a measure of the number of third dredge-up episodes, compared to their $[\mathrm{C} / \mathrm{Fe}]=2.11$. For barium, a predominately $s$-process element, our $[\mathrm{Ba} / \mathrm{Fe}]=1.79$ is close to their $[\mathrm{Ba} / \mathrm{Fe}]=1.93$. The differences between the strontium and the lead abundances are also minor, less than 0.2 dex.

\subsection{Network differences}

We also compare the effect of using an updated reaction network during the post-processing nucleosynthesis calculations. The updated network is based on the JINA Reaclib database as of May 2012 (as compared to the previous network as of May 2009). We perform tests on the $1 \mathrm{M}_{\odot}$ and $3 \mathrm{M}_{\odot}$ stellar models. Figure $3(\mathrm{a})$ shows the results for the $1 \mathrm{M}_{\odot}$ model. There are notable differences in the distribution of the $s$-process elements. The cause of these differences is still to be investigated.

Figure 3(b) shows the results for the $3 \mathrm{M}_{\odot}$ model. The only notable difference is the increased abundance in fluorine when using the updated network. This difference can be explained by the updated ${ }^{19} \mathrm{~F}(\alpha, \mathrm{p})^{22} \mathrm{Ne}$ reaction rate. 


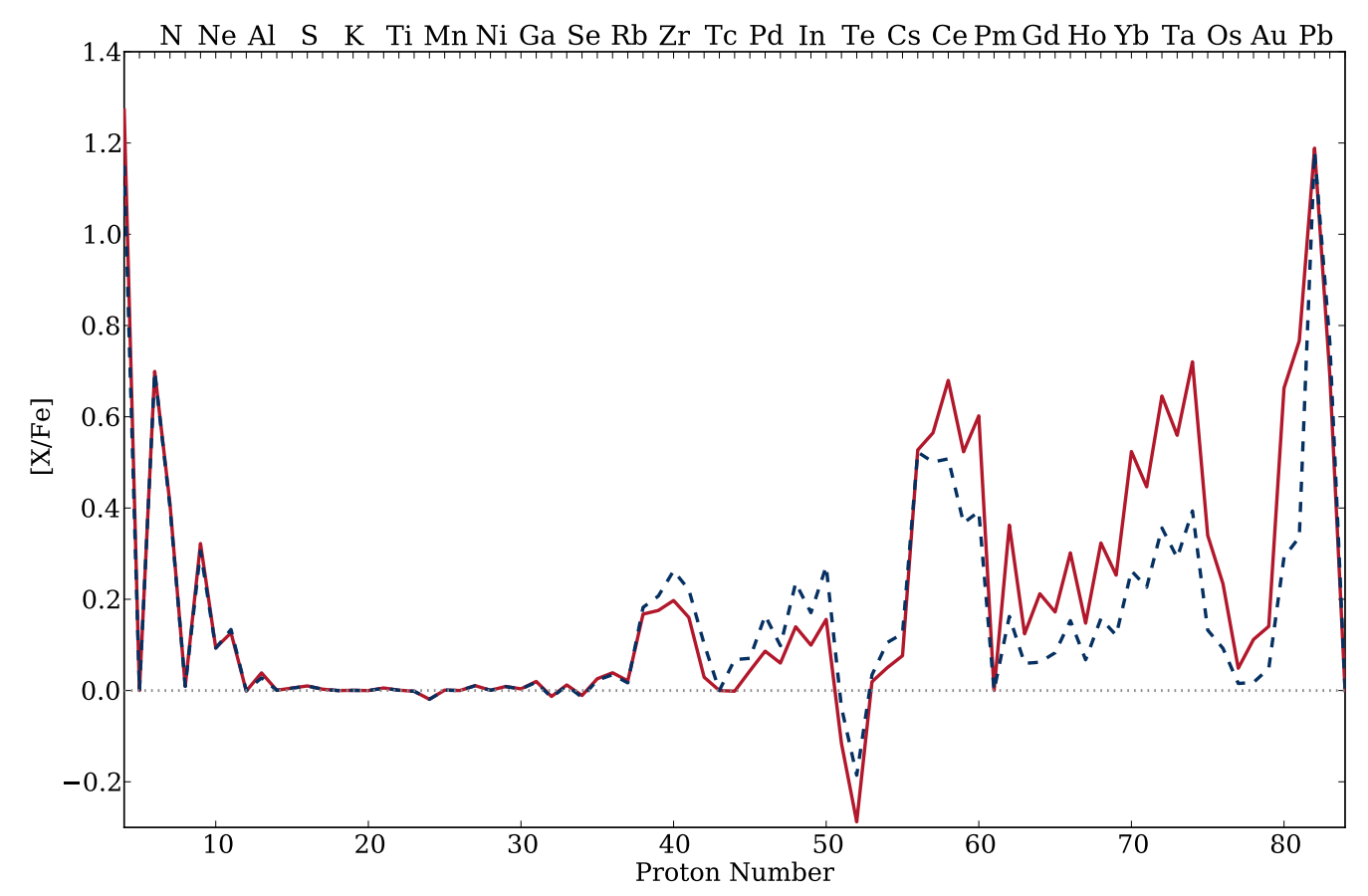

(a) $1 \mathrm{M}_{\odot}$ stellar model of $Z=0.001$.

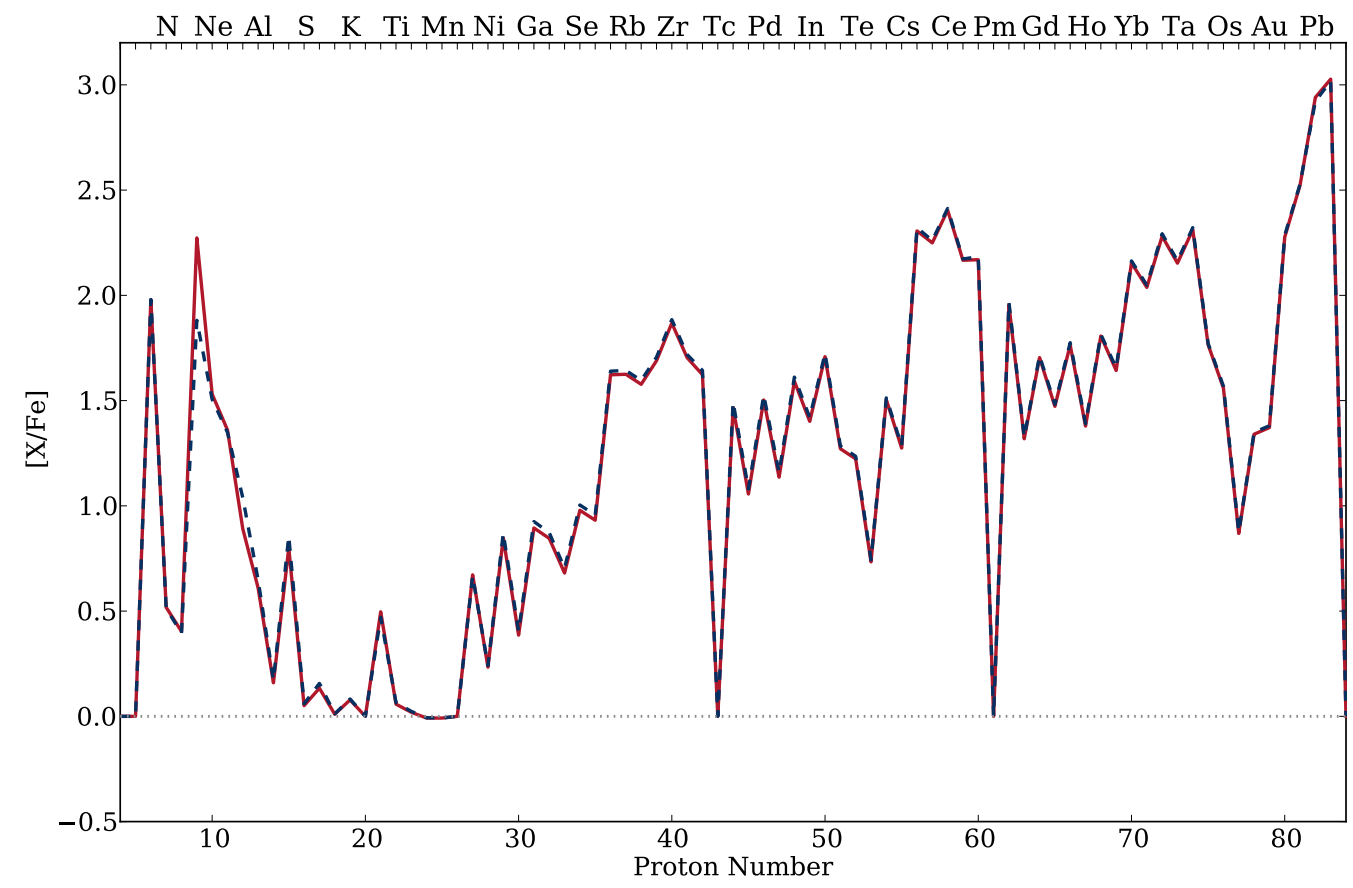

(b) $3 \mathrm{M}_{\odot}$ stellar model of $Z=0.001$.

Figure 3: Comparison of the final surface abundance for two stellar models using the JINA Reaclib database as of 2009 and 2012. The dashed (blue) line shows the results for the 2009 reaction network while the solid (red) line shows the results for the updated 2012 reaction network. 


\section{Conclusion}

Five stellar models have been calculated using the full neutron-capture network $-1 \mathrm{M}_{\odot}, 1.25 \mathrm{M}_{\odot}$, $1.5 \mathrm{M}_{\odot}, 2.5 \mathrm{M}_{\odot}$ and $3 \mathrm{M}_{\odot}$ at a metallicity of $Z=0.001$. These stellar models represent the first in a grid that will span a range of mass $\left(1 \mathrm{M}_{\odot}\right.$ to $\left.6 \mathrm{M}_{\odot}\right)$ and metallicity $([\mathrm{Fe} / \mathrm{H}]$ of $-1.6,-1.2$ and -0.8$)$. A comparison was also made to a $1.5 \mathrm{M}_{\odot}$ stellar model calculated by Cristallo et al. (2011) where the difference in abundances of the neutron-capture peaks were minor. Further details including stellar yields will be presented in a forthcoming paper.

Our intention is to use these models to study the evolution of the Galactic halo and the accretion of dwarf galaxies via the investigation of possible differences in neutron-capture abundances due to the contribution of AGB stars.

\section{References}

[1] Asplund, M., Grevesse, N., Sauval, A. J., \& Scott, P. 2009, Annual Review of Astronomy and Astrophysics, 47, 481

[2] Busso, M., Gallino, R., \& Wasserburg, G. J. 1999, Annual Review of Astronomy and Astrophysics, 37,239

[3] Cristallo, S., Piersanti, L., Straniero, O., Gallino, R., Domìnguez, I., Abia, C., Di Rico, G., Quintini, M., \& Bisterzo, S. 2011, The Astrophysical Journal Supplement, 197, 17

[4] Eggen, O. J., Lynden-Bell, D., \& Sandage, A. R. 1962, Astrophysical Journal, 136, 748

[5] Herwig, F. 2005, Annual Review of Astronomy and Astrophysics, 43, 435

[6] Iglesias, C. A. \& Rogers, F. J. 1996, Astrophysical Journal v.464, 464, 943

[7] Karakas, A. I., Campbell, S. W., \& Stancliffe, R. J. 2010, The Astrophysical Journal, 713, 374

[8] Karakas, A. I., Garcìa-Hernàndez, D. A., \& Lugaro, M. 2012, The Astrophysical Journal, 751, 8

[9] Lodders, K. 2003, The Astrophysical Journal, 591, 1220

[10] Lugaro, M., Karakas, A. I., Stancliffe, R. J., \& Rijs, C. 2012, The Astrophysical Journal, 747, 2

[11] Marigo, P. \& Aringer, B. 2009, Astronomy and Astrophysics, 508, 1539

[12] Nissen, P. E. \& Schuster, W. J. 2010, Astronomy and Astrophysics, 511, L10

[13] Nissen, P. E. \& Schuster, W. J. 2011, Astronomy and Astrophysics, 530, A15

[14] Searle, L. \& Zinn, R. 1978, Astrophysical Journal, 225, 357 